\title{
Modifiable Factors Explain Socioeconomic Inequalities in Children's Dental Caries
}

Journal of Dental Research

I-8

(C) International \& American Associations for Dental Research 2019

Article reuse guidelines: sagepub.com/journals-permissions DOI: $10.1177 / 0022034519866628$ journals.sagepub.com/home/jdr

\author{
M.A. Peres ${ }^{1,2} \mathbb{D}$, X. Ju' ${ }^{\mathbb{D}}$, M. Mittinty ${ }^{3}$, A.J. Spencer', and L.G. Do'
}

\begin{abstract}
The aim of this article was to quantify socioeconomic inequalities in dental caries experience among Australian children and to identify factors that explain area-level socioeconomic inequalities in children's dental caries. We used data from the National Child Oral Health Survey conducted in Australia between 2012 and $2014(n=24,664)$. Absolute and relative indices of socioeconomic inequalities in the dental caries experience in primary and permanent dentition (decayed, missing, and filled surfaces [dmfs] and DMFS, respectively) were estimated. In the first stage, we conducted multilevel negative binomial regressions to test the association between area-level Index of Relative Socioeconomic Advantage and Disadvantage (IRSAD) and dental caries experience (dmfs for 5- to 8-y-olds and DMFS for 9- to I4-y-olds) after adjustment for water fluoridation status, sociodemographics, oral health behaviors, pattern of dental visits, and sugar consumption. In the second stage, we performed Blinder-Oaxaca and Neumark decomposition analyses to identify factors that explain most of the area-level socioeconomic inequalities in dental caries. Children had a mean dmfs of 3.14 and a mean DMFS of 0.98 surfaces. Children living in the most disadvantaged and intermediately disadvantaged areas had 1.96 (95\% confidence interval, 1.69-2.27) and I.45 (I.26-I.68) times higher mean dmfs and I.53 (I.36-I.72) and I.43 (I.27-I.60) times higher mean DMFS than those living in the most advantaged areas, respectively. Water fluoridation status (33.6\%), sugar consumption (22.1\%), parental educational level (I4.2\%), and dental visit patterns (12.7\%) were the main factors explaining area-level socioeconomic inequalities in dental caries in permanent dentition. Among all the factors considered, the factors that contributed most in explaining inequalities in primary dental caries were dental visits (30.3\%), sugar consumption (20.7\%), household income (20.0\%), and water fluoridation status (I5.9\%). The inverse arealevel socioeconomic inequality in dental caries was mainly explained by modifiable risk factors, such as lack of fluoridated water, high sugar consumption, and an unfavorable pattern of dental visits.
\end{abstract}

Keywords: epidemiology, mathematical modelling, statistics, dental public health, oral health, socioeonomic factors

\section{Introduction}

There is criticism that social oral epidemiology has been limited to documenting socioeconomic inequalities and has mostly failed to understand and explain them (Baker and Gibson 2014). Despite agreeing with such criticism, the measurement and documentation of socioeconomic inequalities in oral health by using appropriate and sophisticated techniques have rarely been found in the dental literature (Peres et al. 2015). Measurement and documentation should be considered the first step of any epidemiological study on oral health inequalities. The second step involves the comprehensive appraisal of individual- and area-level factors to understand why variations exist and which factors are associated with oral health outcomes. Finally, as a third step, it is essential to seek accountability for these inequalities. One way is by decomposing socioeconomic inequalities into their contributing factors. This is possible by using approaches such as the Blinder Oaxaca technique. Applying this technique, it is possible to examine the sources of, for example, caries difference between high and low socioeconomic groups by constructing a counterfactual equation where people from the low socioeconomic group are treated as those in the high socioeconomic group. To do that, the intercept and coefficient in the low socioeconomic group equation are replaced by those of the high socioeconomic group. Caries difference between socioeconomic groups can then be decomposed into different factors such as sugar intake and fluoride, among others.

This approach is even rarer in the dental literature (AyoYusuf et al. 2013; Safiri et al. 2016; Li et al. 2018). The full description, understanding, and explanation of which potentially modifiable factors matter may help us to infer how policies would affect socioeconomic inequality. Modifiable risk factor is a determinant that can be modified by intervention, thereby reducing the probability of disease (Burt 2001).

\footnotetext{
'Australian Research Centre for Population Oral Health (ARCPOH), Adelaide Dental School, The University of Adelaide, Adelaide, Australia ${ }^{2}$ Menzies Health Institute Queensland and School of Dentistry and Oral Health, Griffith University, Gold Coast, Australia

${ }^{3}$ School of Public Health, The University of Adelaide, Adelaide, Australia

A supplemental appendix to this article is available online.

\section{Corresponding Author:}

M.A. Peres, Menzies Health Institute Queensland and School of Dentistry and Oral Health, Griffith University, Griffith Health Centre (G40, Level 7), room 7.58, Gold Coast Campus, Queensland 4222, Australia.

Email: m.peres@griffith.edu.au
} 
There is a gap in information regarding area-level socioeconomic inequalities in children's dental caries in Australia. The country has a high proportion of people covered by water fluoridation, nearly $90 \%$ of the population. However, the dental care system was accessible for less than $50 \%$ of children aged 2 to 17 y between 2014 and 2016, even after the implementation of a federal child dental care program, the Child Dental Benefit Schedule, which was designed to cover $70 \%$ of the child population.

From a policymaking perspective, along with denouncing unfair and unjust inequalities, it is essential to develop and implement effective measures to tackle such inequalities.

Therefore, the aims of this study are to measure relative and absolute socioeconomic inequalities in children's dental caries experience, estimate whether socioeconomic inequalities at the area level persist after adjustment for individual-level factors, and quantify how much of the difference in socioeconomic area level is explained by individual-level factors.

\section{Methods}

This study is reported according to Strengthening the Reporting of Observational Studies in Epidemiology guidelines.

\section{Setting and Sampling}

The National Child Oral Health Survey (NCOHS) 2012-14 was a nationwide Australian survey. The target population was children aged 5 to $14 \mathrm{y}$, and the total sample size was 24,664 children. Primary and secondary schools were included in the sampling frame. A sampling frame of schools was created from a list provided by each jurisdiction that included all public, Catholic, and independent primary and secondary schools. Information provided on the sampling frame for each school included school identification number, school name and address, school type, school enrollment, and health district. The sampling frame was first stratified by geographical region and then by primary versus secondary/combined school and then sorted by a composite index of either school or area socioeconomic status. Schools were then selected with a probability proportional to size of enrollment. In the second stage, children were randomly sampled from each participating school. In total, 841 schools consented to participate out of 1,250 schools invited. Detailed aspects of the methodology of the study have been published elsewhere (Do and Spencer 2016).

\section{Outcomes}

Dental examination included caries assessments at the toothsurface level. We used disease experience expressed as the mean number of decayed $(\mathrm{d} / \mathrm{D})$, missing $(\mathrm{m} / \mathrm{M})$, and filled $(\mathrm{f} / \mathrm{F})$ surfaces, which allowed us to estimate dmfs and DMFS for primary and permanent dentitions, respectively. The criteria and methods for the assessment of caries experience were based on the US National Health and Nutrition Examination Survey (Centers for Disease Control and Prevention [CDC] 2009-2010). A total of 62 dental examiners collected the data.
Testing interexaminer reliability among examiners was not logistically and financially practical because of the spread of examiners across the whole country. Also, states/territories conducted the fieldwork at different times. More important, testing reliability between examiners and the trainers ensured examiners' adherence to the examination protocol. That adherence reduced examiner-related variation in collected data across geographical locations. All examiners were tested in the field against 1 of 2 senior trainers to estimate interexaminer reliability. This method has been used in US NHANES surveys and the UK Dental Health Surveys. The intraclass correlation coefficients (ICCs) for caries experience scores for dmfs for 5- to 8-y-olds and DMFS for 9- to 14-y-olds were 0.95 and 0.69 , respectively, indicating moderate to excellent reliability (Spencer et al. 2018).

\section{Explanatory Variables}

A questionnaire was given to the participants' parents, including information about sources of exposure to fluoride, dietary intake, child's health, use and evaluation of dental services, birthplace, and household characteristics.

For descriptive purposes, we used 4 area- and individuallevel socioeconomic indicators to assess socioeconomic inequalities in dental caries. Parental educational level and household income were used at the individual level. At the area level, we used the Index of Relative Socioeconomic Advantage and Disadvantage (IRSAD) and Index of Community SocioEducational Advantage (ICSEA). The IRSAD summarizes information about the economic and social conditions of people and households within an area, including both relative advantage and disadvantage measures (Australia Bureau of Statistics [ABS] 2001). The ICSEA is an index that combines students' household characteristics (such as parental occupation and level of education) and school area characteristics, such as the proportion of indigenous and geographical location (Australian Curriculum, Assessment and Reporting Authority [ACARA] 2013). Parental education was categorized into school, vocational training, and university; annual household income was categorized into 3 groups: low ( $<$ AU\$60,000.00), medium (AU\$60,000.00 to AU\$120,000.00), and high (>AU\$120,000.00). IRSAD and ICSEA were divided into tertiles.

Individual-level explanatory variables included sex (boy/ girl), current toothbrushing with fluoride toothpaste (>once a day/once a day or less), last dental visit (checkup/problem), fluoride concentration in the water $(\geq 0.6 /<0.6 \mathrm{ppm} \mathrm{F} / \mathrm{L})$, and sugar-sweetened beverage (SSB) consumption per day $(0,1$, and $\geq 2$ medium glasses). Information on SSBs was collected using a list of common beverages. Separate questions were used for sweetened or unsweetened beverages in each group. Examples were provided.

\section{Data Analyses}

The mean dmfs and DMFS were calculated according to each explanatory variable. We drew equiplot graphics that provide 
the distance between socioeconomic groups (household income, parental education, IRSAD, and ICSEA). We assessed absolute and relative socioeconomic inequalities on children's dental caries by calculating the Slope Index of Inequality (SII) and the Relative Index (RI) of inequality (Mackenbach and Kunst 1997). Negative values of SII and RI indicate concentration of the disease (dmfs/DMFS) among the lower socioeconomic status group. We performed multilevel negative binomial regressions to test the association between area-level IRSAD and dental caries experience (dmfs for 5-8 y and DMFS for 9-14 y) after adjustment for water fluoridation status, sociodemographics, toothbrushing with fluoride toothpaste, dental visits, and sugar consumption. The estimated association measure was the dmfs or DMFS mean ratio (MR) and its $95 \%$ confidence intervals (CIs). Variance estimates at the area level were calculated by means of median rate ratio (MRR) and proportion change in variance (PCV) (Larsen and Merlo 2005; Merlo et al. 2006). Given the strong collinearity between IRSAD and ICSEA, the latter was not used in analyses while parental education and household income were entered in the multivariable multilevel analyses.

To identify the factors that explain most of the area-level socioeconomic inequalities in dental caries, we performed Blinder-Oaxaca and Neumark decomposition analyses. This counterfactual approach allows a comparison between what actually happened and what would have happened in the absence of the intervention. Impact evaluations seek to answer cause-and-effect questions. In other words, they look for the changes in outcome that are directly attributable to a specific intervention or policy.

Area-level IRSAD was recategorized into 2 levels: lowlowest IRSAD versus high-medium plus highest IRSAD. Decomposition methods reveal the extent to which inequalities in health can be explained by a set of factors that vary systematically with area-level socioeconomic status. The BlinderOaxaca decomposition is a counterfactual analysis that explains the change in the mean values of the potential outcome (dmfs and DMFS) when the individual in the data is set to have a low IRSAD and the same individual is set to be in the high IRSAD. Furthermore, it allows for the decomposition of these mean differences into that explained by the explanatory variable (Blinder 1973; Oaxaca 1973). All coefficients were obtained from the pooled data regression (Neumark 1988). All analyses were conducted using the oxaca command in Stata 13. Detailed formulas and computational coding are presented as online supplementary material.

\section{Ethical Issues}

NCOHS was approved by University of Adelaide Human Research Ethics Committee. Parents provided signed, informed consent for their child to participate.

\section{Results}

A total of 24,664 children participated in NCOHS. Table 1 describes the sample characteristics. Overall, 2 in 10 children had their last dental visit for a dental problem, 3 in 10 brushed their teeth up to once a day, 3 in 10 lived in an area with low fluoride concentration in the water, and almost one-quarter drank 2 or more medium glasses of SSBs a day. Slightly less than one-quarter of the children had parents with a school education only, and almost half of them had university-educated parents. Almost one-third of the sample lived in a household with an income of less than $\$ 60,000$ a year. Nearly $30 \%$ of the children were enrolled in schools with ICSEA scores below the national average; approximately $30 \%$ of the children lived in areas with an IRSAD level below the national average.

Children had a mean dmfs of 3.14 and a mean DMFS of 0.98 surfaces (Table 1). The Figure presents the mean dmfs of children aged 5 to 8 y by socioeconomic indicators. The distances between the mean dental caries experience between the medium and the lowest socioeconomic groups are larger than between the medium and the highest socioeconomic groups. The Appendix Figure shows the average DMFS of children 9 to $14 \mathrm{y}$ according to socioeconomic indicators.

All measures of absolute and relative socioeconomic inequalities were negative, which means that from the bottom to the top of the socioeconomic ladder, dental caries experience decreased. The highest absolute socioeconomic inequality measure was with ICSEA, but all 4 indicators (IRSAD, ICSEA, household income, and parental education) had a statistically similar magnitude. Similar results occurred for the measures of relative socioeconomic inequalities (Appendix Table 1).

There was a clear gradient in dental caries experience in primary and permanent dentitions across the IRSAD levels. The most disadvantaged area had the highest average dmfs and DMFS (Table 2, model 2). After adjustment for oral healthrelated behaviors, the association between experience of dental caries and area-level socioeconomic indicators decreased (Table 2, model 5). The magnitudes of MRRs for all primary and permanent dentition multilevel models were similar, between 2.26 and 2.64. However, PCV values were higher for primary dentition than those for permanent dentition, reaching $11.73 \%$ in the fully adjusted model.

Table 3 shows the decomposition analyses of contributors to the area-level IRSAD effect on the observed gap in dental caries experience in the primary and permanent dentitions. The proportion explained is largely related to change in endowments, attributable to IRSAD level changes in the magnitude of the explanatory variables.

The modifiable factors that explained such inequalities in primary dental caries were dental visits $(30.3 \%)$, sugar consumption (20.7\%), and water fluoridation status (15.9\%). Water fluoridation status (33.6\%), sugar consumption (22.1\%), and dental visits $(12.7 \%)$ were the main modifiable factors that explain the caries difference between the low-lowest IRSAD versus the highmedium plus highest IRSAD in permanent dentition.

\section{Discussion}

There are consistent area-level socioeconomic inequalities in children's dental caries in Australia. Socioeconomic inequalities are more pronounced in primary than in permanent 
Table I. Sample Characteristics among Australian Children (Weighted).

\begin{tabular}{|c|c|c|c|c|c|c|}
\hline \multirow[b]{2}{*}{ Characteristic } & \multicolumn{2}{|c|}{ All Ages } & \multicolumn{2}{|c|}{ Aged $5-8$ y } & \multicolumn{2}{|c|}{ Aged 9-14 y } \\
\hline & $n$ & $\%(95 \% \mathrm{Cl})$ & $n$ & $\%(95 \% \mathrm{Cl})$ & $n$ & $\%(95 \% \mathrm{Cl})$ \\
\hline All & 24,664 & - & 10,599 & $40.2(39.3-4 I .1)$ & 14,065 & $59.8(58.9-60.7)$ \\
\hline \multicolumn{7}{|l|}{ Sex } \\
\hline Boy & 12,348 & $51.2(50.3-52.2)$ & 5,259 & $51.9(50.5-53.3)$ & 7,089 & $50.8(49.6-52.0)$ \\
\hline Girl & 12,316 & $48.8(47.8-49.7)$ & 5,340 & $48.1(46.7-49.5)$ & 6,976 & $49.2(48.0-50.4)$ \\
\hline \multicolumn{7}{|l|}{ Dental visiting } \\
\hline Checkup & 16,962 & $80.2(79.4-81.0)$ & 6,528 & $78.5(77.1-79.8)$ & 10,434 & $81.1(80.1-82.1)$ \\
\hline Problem & 4,075 & $19.8(19.0-20.6)$ & 1,623 & $21.5(20.2-22.9)$ & 2,452 & $18.9(17.9-19.9)$ \\
\hline \multicolumn{7}{|l|}{ Toothbrushing } \\
\hline$>$ I/d & 17,134 & $68.5(67.6-69.4)$ & 7,278 & $67.4(66.0-68.8)$ & 9,856 & $69.2(29.6-31.9)$ \\
\hline$\leq \mathrm{I} / \mathrm{d}$ & 7,077 & $31.5(30.6-32.4)$ & 3,139 & $32.6(31.2-34.0)$ & 3,938 & $30.8(68.1-70.4)$ \\
\hline \multicolumn{7}{|l|}{ Sugar consumption (medium glass) } \\
\hline$\geq 2$ & 4,530 & $24.7(23.8-25.6)$ & 1,530 & $19.5(\mid 8.3-20.7)$ & 3,000 & $28.2(27.0-29.4)$ \\
\hline I & 6,607 & $31.1(30.2-32.1)$ & 2,615 & $29.0(27.6-30.4)$ & 3,992 & $32.6(31.4-33.8)$ \\
\hline 0 & 10,375 & $44.2(43.2-45.2)$ & 5,094 & $51.6(50.0-53.1)$ & 5,281 & $39.3(38.0-40.5)$ \\
\hline \multicolumn{7}{|l|}{ Fluoride concentration (ppm) } \\
\hline$\geq 0.6$ & 16,053 & $69.0(68.2-69.8)$ & 7,017 & $69.6(68.3-70.8)$ & 9,036 & $68.6(67.6-69.7)$ \\
\hline $0-0.6$ & 7,930 & $31.0(30.2-31.8)$ & 3,281 & $30.4(29.2-31.7)$ & 4,649 & $31.4(30.3-32.4)$ \\
\hline \multicolumn{7}{|l|}{ Parental education } \\
\hline EDU I (lowest: school) & 4,767 & $28.9(28.0-29.8)$ & 1,908 & $27.8(26.4-29.2)$ & 2,859 & $29.6(28.4-30.8)$ \\
\hline EDU 2 (medium: vocational training) & 4,213 & $22.5(21.7-23.3)$ & 1,713 & $21.5(20.3-22.8)$ & 2,500 & $23.1(22.1-24.2)$ \\
\hline EDU 3 (highest: university) & 14,160 & $48.6(47.7-49.6)$ & 6,269 & $50.6(49.2-52.1)$ & 7,891 & $47.3(46.0-48.5)$ \\
\hline \multicolumn{7}{|l|}{ Household income } \\
\hline Income I (lowest: $<\$ 60,000)$ & 6,988 & $32.5(31.6-33.4)$ & 2,902 & $31.6(30.3-33.0)$ & 4,086 & $33.1(31.9-34.3)$ \\
\hline Income 2 (medium: $\$ 60,000$ to $\$ 120,000$ ) & 9,755 & $38.4(37.5-39.3)$ & 4,275 & $37.8(36.4-39.2)$ & 5,480 & $38.8(37.6-40.0)$ \\
\hline Income 3 (highest: $>\$ 120,000$ ) & 6,643 & $29.1(28.2-30.0)$ & 2,377 & $30.6(29.1-32.0)$ & 3,653 & $28.1(27.0-29.3)$ \\
\hline \multicolumn{7}{|l|}{ Area level (IRSAD) } \\
\hline IRSAD I (lowest: IRSAD score <948) & 8,004 & $28.4(27.6-29.2$ & 3,377 & $28.8(27.5-30.0)$ & 4,627 & $28.1(27.1-29.2)$ \\
\hline IRSAD 2 (medium: IRSAD score 948-I,026) & 8,343 & $37.6(36.7-38.5$ & 3,625 & $38.2(36.8-39.5)$ & 4,718 & $37.3(36.1-38.4)$ \\
\hline IRSAD 3 (highest: IRSAD score > I,026) & 8,292 & $34.0(33.1-34.9$ & 3,585 & $33.1(31.7-34.5)$ & 4,707 & $34.6(33.4-35.8)$ \\
\hline \multicolumn{7}{|l|}{ School level (ICSEA) } \\
\hline ICSEA I (lowest: ICSEA score <986) & 8,238 & $34.0(33.1-34.9)$ & 3,459 & $33.6(32.3-34.9)$ & 4,779 & $34.3(33.1-35.4)$ \\
\hline ICSEA 2 (medium: ICSEA score 986-I,044) & 8,201 & $30.9(30.1-3 \mid .8)$ & 3,560 & $31.3(30.0-32.6)$ & 4,641 & $30.7(29.6-31.8)$ \\
\hline \multirow[t]{2}{*}{ ICSEA 3 (highest: ICSEA score >I,044) } & 8,225 & $35.1(34.2-36.0)$ & 3,580 & $35.2(33.8-36.6)$ & 4,645 & $35.0(33.9-36.2)$ \\
\hline & & & & Mean $(95 \% \mathrm{Cl})$ & & Mean $(95 \% \mathrm{Cl})$ \\
\hline dmfs & & & & $3.14(3.02-3.26)$ & & \\
\hline DMFS & & & & & & $0.98(0.95-1.02)$ \\
\hline
\end{tabular}

dmfs, decayed, missing, and filled surfaces for primary dentition; DMFS, decayed, missing, and filled surfaces for permanent dentition; ICSEA, Index of Community Socio-Educational Advantage; IRSAD, Index of Relative Socioeconomic Advantage and Disadvantage.

dentition. Altogether, modifiable factors, such as dental visits, toothbrushing, sugar consumption, and fluoride concentration in the water, explained $64.3 \%$ and $70.4 \%$ of the total area-level socioeconomic inequalities in primary and permanent children's caries, respectively.

These findings suggest there are opportunities for policymakers to develop and implement policies to reduce these gaps by reducing sugar intake, increasing water fluoridation coverage, and moving dental visits toward a favorable pattern. Most of these policies should be upstream and call for intersectoral and multidisciplinary approaches. What should be done?

\section{Restriction of Sugar Intake}

Our findings revealed that SSB consumption explained slightly over one-fourth of the area-level income inequality in both primary and permanent dentition caries. More structural policies, such as less use of sugars at the national level by reformulation of food products and reducing their serve sizes, as well as introduction of taxation of sugar products, have been recommended. On the other hand, downstream measures include the improvement of education to health professionals (Moynihan et al. 2018). The World Health Organization (WHO) recommends that free sugars intake should be restricted to less than $10 \%$ of total energy, highlighting that for further benefits, restriction in sugar consumption should be up to $5 \%$ of total energy. However, Australia is far away from achieving these goals (Wittekind and Walton 2014; ABS 2016).

There is a debate in Australia regarding the effectiveness and appropriateness of having a "sugar tax." Many public health, academic, and consumer groups support a tax on SSBs. However, the political support is limited, and there is a strong lobby against the implementation of an SSB tax led by sugary drinks companies (Backholer and Martin 2017). 

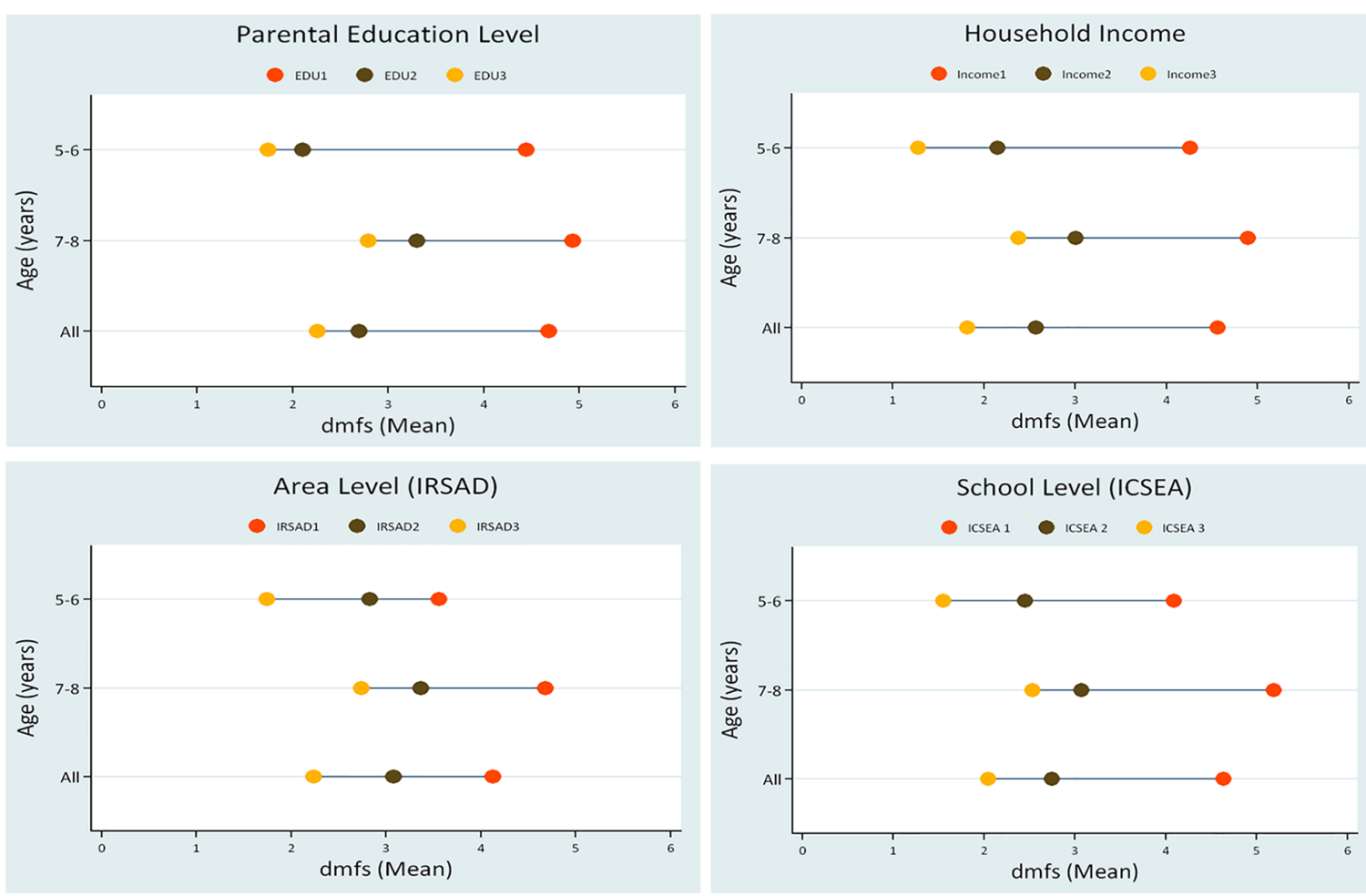

University of Adelaide

Figure. Average number of decayed, missing, or filled tooth surfaces (dmfs) of the primary dentition according to socioeconomic characteristics. ICSEA, Index of Community Socio-Educational Advantage; IRSAD, Index of Relative Socioeconomic Advantage and Disadvantage.

Table 2. Mean Ratio and $95 \% \mathrm{Cl}$ of Dental Caries Experience (dmfs/DMFS) among Australian Children (Multilevel Analyses).

\begin{tabular}{|c|c|c|c|c|c|}
\hline \multirow[b]{2}{*}{ Characteristic } & \multicolumn{5}{|c|}{ Mean Ratio $(95 \% \mathrm{Cl})$} \\
\hline & Model I & Model 2 & Model 3 & Model 4 & Model 5 \\
\hline \multicolumn{6}{|l|}{ Aged 5-8 y (dmfs) } \\
\hline \multicolumn{6}{|l|}{ Area level } \\
\hline IRSAD I & & $1.96(1.69-2.27)$ & $1.82(1.56-2.11)$ & $1.30(1.10-1.53)$ & I.IS (0.96-I.38) \\
\hline IRSAD 2 & & $1.45(1.26-1.68)$ & $1.36(1.18-1.58)$ & $1.08(0.93-1.25)$ & $1.03(0.88-1.21)$ \\
\hline IRSAD 3 & & 1.00 & 1.00 & 1.00 & 1.00 \\
\hline \multicolumn{6}{|c|}{ Level 2 variance } \\
\hline MRR & $2.64(1.60-5.02)$ & $2.55(1.50-4.62)$ & $2.48(1.43-4.37)$ & $2.26(1.22-3.60)$ & $2.35(1.31-3.83)$ \\
\hline PCV (\%) & & 3.26 & 4.59 & 9.50 & 11.73 \\
\hline \multicolumn{6}{|c|}{ Aged 9-I4 y (DMFS) } \\
\hline \multicolumn{6}{|c|}{ Area level } \\
\hline IRSAD I & & I.53 (I.36-I.72) & $1.36(1.21-1.54)$ & 1.14 (1.00-1.30) & $1.03(0.89-1.18)$ \\
\hline IRSAD 2 & & $1.43(1.27-1.60)$ & 1.31 (1.17-1.47) & 1.17 (1.03-1.32) & $1.12(0.98-1.27)$ \\
\hline IRSAD 3 & & 1.00 & 1.00 & 1.00 & 1.00 \\
\hline \multicolumn{6}{|c|}{ Level 2 variance } \\
\hline MRR & $2.45(1.5 \mathrm{I}-3.94)$ & $2.44(1.50-3.90)$ & $2.39(1.44-3.70)$ & $2.38(1.43-3.73)$ & $2.32(1.34-3.57)$ \\
\hline PCV (\%) & & 0.50 & 3.68 & 0.77 & -4.21 \\
\hline
\end{tabular}

Model I: is an intercept-only (null) model adjusted for child's sex and mean center of age. Model 2: model I plus area level (IRSAD). Model 3: model 2 plus fluoride concentration. Model 4: model 3 plus parental education level and household income. Model 5: model 4 plus dental visit for a checkup,

toothbrushing, and sugar-sweetened beverage consumption. $M R R=\exp (0.95 * \sqrt{\text { Area }}$ variance $) . P C V=\frac{V_{\text {Modell }}-V_{\text {Modeln }}}{V_{\text {Modell }}}$, where $V_{\text {Modell }}=$ variance of model I, $V_{\text {odel }}=$ variance of $n$, and $n=2-6$. Mean center of age $=$ mean - age.

$\mathrm{dmfs}$, decayed, missing, and filled surfaces for primary dentition; DMFS, decayed, missing, and filled surfaces for permanent dentition; ICSEA, Index of Community Socio-Educational Advantage; IRSAD, Index of Relative Socioeconomic Advantage and Disadvantage; MRR, median rate ratio; PCV, proportional change in variance. 
Table 3. Decomposition of Contributors to Area-Level (Lowest vs. Medium + Highest) Effect on Observed Gap in Dental Caries Experience (dmfs/DMFS) among Australian Children.

\begin{tabular}{|c|c|c|c|c|c|c|c|c|}
\hline Characteristic & \multicolumn{4}{|c|}{ Aged 5-8 y } & \multicolumn{4}{|c|}{ Aged 9-14 y } \\
\hline Mean dmfs/DMFS (lowest) & \multicolumn{4}{|c|}{4.485} & \multicolumn{4}{|c|}{1.023} \\
\hline $\begin{array}{l}\text { Mean dmfs/DMFS (medium } \\
+ \text { highest) }\end{array}$ & \multicolumn{4}{|c|}{2.811} & \multicolumn{4}{|c|}{0.884} \\
\hline $\begin{array}{l}\text { Raw difference between } 2 \\
\text { area levels }\end{array}$ & \multicolumn{4}{|c|}{1.674} & \multicolumn{4}{|c|}{0.140} \\
\hline Due to endowments $(E)$ & \multicolumn{4}{|c|}{1.296} & \multicolumn{4}{|c|}{0.253} \\
\hline Due to coefficients $(\mathrm{C})$ & \multicolumn{4}{|c|}{$\begin{array}{l}0.203 \\
0.175\end{array}$} & \multicolumn{4}{|c|}{-0.166} \\
\hline Due to interaction (CE) & \multicolumn{4}{|c|}{0.175} & \multicolumn{4}{|c|}{0.052} \\
\hline Explanatory Variables & $E(D=0)$ & C & E (Neumark) & $\begin{array}{c}\text { Proportion } \\
\text { Explained (\%) }\end{array}$ & $E(D=0)$ & C & E (Neumark) & $\begin{array}{c}\text { Proportion } \\
\text { Explained (\%) }\end{array}$ \\
\hline Sex & 0.001 & -0.178 & 0.002 & 0.15 & -0.000 & 0.012 & -0.000 & 0.000 \\
\hline Dental visiting & 0.410 & -0.036 & 0.415 & 30.25 & 0.026 & -0.271 & 0.032 & 12.65 \\
\hline Toothbrushing & 0.005 & -0.896 & 0.014 & 1.02 & 0.020 & 0.076 & 0.016 & 6.32 \\
\hline Sugar consumption & 0.267 & -0.206 & 0.284 & 20.70 & 0.049 & -0.153 & 0.056 & 22.13 \\
\hline Fluoride concentration & 0.195 & -0.204 & 0.218 & 15.89 & 0.093 & 0.012 & 0.085 & 33.60 \\
\hline Parental education level & 0.126 & -0.594 & 0.164 & 11.95 & 0.051 & 0.181 & 0.036 & 14.23 \\
\hline Household income & 0.292 & 0.279 & 0.275 & 20.04 & 0.016 & -0.189 & 0.028 & II.07 \\
\hline Explained $(\mathrm{V})\{\mathrm{E}+\mathrm{D} * \mathrm{CE}\}$ & 1.296 & 0.203 & $\mathrm{I} .372$ & 100.00 & 0.253 & -0.166 & 0.253 & 100.00 \\
\hline
\end{tabular}

The coefficients were obtained from the pooled data regression. E (and E Neumark), C, and CE show the contribution attributable to the gaps in endowments $(E)$, the coefficients $(C)$, and due to the interaction (CE). In this study, the gap in endowments accounts for the great bulk of the gap in outcomes. Proportion explained: related to change in endowments, attributable to IRSAD level changes in the magnitude of the explanatory variables. Unexplained: related to change in coefficients.

dmfs, decayed, missing, and filled surfaces for primary dentition; DMFS, decayed, missing, and filled surfaces for permanent dentition; IRSAD, Index of Relative Socioeconomic Advantage and Disadvantage.

There is no evidence of the long-term effect of most studied interventions to reduce the amount of sugar consumption (Kirkpatrick et al. 2018). However, most of the research was undertaken in a context-specific social, cultural, and political environment, mainly in the United States. Therefore, it is difficult to generalize their findings. Moreover, there is a strong focus on sugar and SSBs, minimizing the fact that people are exposed to free sugar as well. SSBs will become less relevant as a source of sugar because of taxation given the potential consumers' shift to other sugar products. This suggests that the focus should be broader than SSB taxation only.

Promisingly, a study suggests that interventions would be more beneficial for economically disadvantaged people (Kirkpatrick et al. 2018), which has been found also in Australia (Lal et al. 2017). In addition, there is moderate-quality evidence that caries is reduced when free-sugar intake is less than $10 \%$ of the total energy intake. However, the evidence of the reduction in caries under scenarios when sugar intake is limited to less than $5 \%$ is of very poor quality (Moynihan and Kelly 2014). Schwendicke et al. (2016) conducted the only study that evaluated the role of SSB taxes on caries, concluding that implementing a $20 \%$ tax on SSBs is likely to reduce caries increment. However, as in our study, Schwendicke et al. (2016) only assessed SSB consumption, which is a clear limitation.

A comprehensive study performed in Australia showed that the combination of sugar tax and subsidies on fruits and vegetables produces an improvement in population's health (Cobiac et al. 2017) and should be considered from a common risk factor approach.
Radical changes in tackling sugar intake are necessary. One interesting example comes from New Zealand, where the Ministry of Health encourages schools to adopt water-only policies, resulting in absolute restriction of sugar-sweetened beverages, to create health food environments at schools (Ministry of Health 2015). However, there is no research on the long-term beneficial effect of this policy.

\section{Enlargement of Water Fluoridation Coverage}

Our study shows that low levels of fluoride concentration in the water to prevent dental caries explained nearly $15 \%$ and one-third of the area-level income inequality in caries in deciduous and permanent dentition, respectively. Australia has already one of the highest coverage rates of water fluoridation in the world. There was a substantial increase in coverage at the time of NCOHS, and the coverage now accounts for nearly $90 \%$ of the population (National Health and Medical Research Council [NHMRC] 2017). Australian studies on the costeffectiveness of water fluoridation have found that for every dollar spent on fluoridation, between $\$ 7$ and $\$ 18$ is saved in avoided treatment costs (NHMRC 2017). Extending the coverage of water fluoridation (WF) to smaller Australian communities revealed less favorable cost-effectiveness than to communities with at least 1,000 people. However, the implementation of WF even in small communities has been recommended given the substantial dental health inequalities for more remote and regional communities (Cobiac and Vos 2012). Based on our findings and recent evidence, there is an opportunity to 
maximize the benefit of WF in the country by implementing this policy in small, remote, rural areas.

\section{Improving the Pattern of Dental Visits}

Inadequate pattern of dental visiting explained almost onethird and $13 \%$ of the area-level income inequality in dental caries in primary and permanent dentition, respectively. The pattern of dental visits, whether for checkups or problems, affects the magnitude of untreated dental caries. Data not shown revealed that the magnitude of socioeconomic inequalities is higher for untreated dental caries $(d>0)$ than for the prevalence of caries $(\mathrm{dmfs}>0)$, particularly in primary dentition. Perhaps this is because, to some extent, all jurisdictions in Australia have a school dental program without a counterpart for preschool children. This explanation is even reinforced by the fact that the proportion of children aged 5 to $14 \mathrm{y}$ who usually have an irregular dental visiting pattern decreases with age (Do and Spencer 2016). The challenge is to deliver preventive dental care for children under school age. The Australian government implemented the Child Dental Benefit Schedule (CDBS) in 2014 to provide financial support for basic dental care for nearly 3 million children aged 2 to $17 \mathrm{y}$. The CDBS has a cap of $\$ 1,000$ per child over 2 consecutive years (Australian Government Department of Health 2018). However, to our knowledge, there is no study evaluating the cost-effectiveness of the CDBS.

This study has many strengths and some limitations. The strengths of this study include a large representative sample of Australian children, the use of comprehensive questionnaires to collect the social data, the adequate examiner reliability measures for caries measures, the use of sophisticated socioeconomic inequalities measures, and the undertaking of a multilevel analysis taking individual and contextual socioeconomic factors into account as recommended (Singh et al. 2018). In addition to that, we performed decomposition analyses, which are rarely found in dental literature but increasingly used in public health (Lauridsen et al. 2006; Sözmen et al. 2012; Amroussia et al. 2017). The main limitation of the study was the cross-sectional design. The present estimate likely overestimates the true causal effect of problem-oriented dental visits on caries inequalities. While the present analysis assumed a causal framework in which dental visit pattern explains caries occurrence, in the cross-sectional data, some of the observed association is likely a result of caries disease causing a family to seek out restorative dental care. Another limitation of this study is that sugar intake measurement was limited to the number of servings of sweet drinks a day.

The findings of this study have practical implications for the dental profession and policymakers. Most factors that explain socioeconomic inequalities in caries among Australian children are modifiable, such as sugar intake and water fluoridation. However, the feasibility and cost-effectiveness of interventions to tackle these factors should be carefully scrutinized. Sugar intake is a common risk factor for caries and systemic diseases, such as obesity and diabetes, and should be faced by an integrated and multidisciplinary approach. The implementation of water fluoridation in small communities should be considered to tackle socioeconomic inequalities in children's caries. The implementation of universal dental care coverage has been advocated to reduce socioeconomic inequalities in oral health (Mathur et al. 2015) and should be considered in Australia.

Based on our findings and the cumulative available body of evidence, we recommend all the above listed interventions to reduce socioeconomic inequalities in children's dental caries. We will monitor the impact of these interventions along with the economic analysis of their cost-effectiveness.

\section{Author Contributions}

M.A. Peres, contributed to conception, design, data analysis and interpretation, drafted and critically revised the manuscript; X. Ju, M. Mittinty, contributed to design, data analysis and interpretation, drafted and critically revised the manuscript; A.J. Spencer, contributed to design, data acquisition and interpretation, drafted and critically revised the manuscript; L.G. Do, contributed to design, data acquisition, analysis, and interpretation, drafted and critically revised the manuscript. All authors gave final approval and agree to be accountable for all aspects of the work.

\section{Acknowledgments}

The National Child Oral Health Study (NCOHS) 2012-2014 was supported by National Health and Medical Research Council Partnership Grant APP1016326 and in-kind contribution from Australian state/territory health authorities. The study participants, their parents/guardians, and research team members are acknowledged for their contribution. The authors declare no potential conflicts of interest with respect to the authorship and/or publication of this article.

\section{ORCID iDs}

M.A. Peres iD https://orcid.org/0000-0002-8329-2808

X. Ju iD https://orcid.org/0000-0003-4759-3918

\section{References}

Amroussia N, Gustafsson PE, Mosquera PA. 2017. Explaining mental health inequalities in northern Sweden: a decomposition analysis. Glob Health Action. 10(1):1305814.

Australian Bureau of Statistics (ABS). 2001. Census basic community profile and snapshot. Canberra, Australia: ABS.

Australian Bureau of Statistics (ABS). 2016. Australian health survey: consumption of added sugars. Canberra, Australia: ABS.

Australian Curriculum Assessment and Reporting Authority (ACARA). 2013 Guide to understanding 2013 Index of Community Socio-educational Advantage (ICSEA) values. Canberra, Australia: ACARA.

Australian Government Department of Health. 2018. Canberra (Australia): Commonwealth of Australia; [updated 2018 Jan 1]. https:/www1.health .gov.au/internet/main/publishing.nsf/Content/childdental.

Ayo-Yusuf IJ, Ayo-Yusuf OA, Olutola BG. 2013. Health insurance, socioeconomic position and racial disparities in preventive dental visits in South Africa. Int J Environ Res Public Health. 10(1):178-191.

Backholer K, Martin J. 2017. Sugar-sweetened beverage tax: the inconvenient truths. Public Health Nutr. 20(18):3225-3227.

Baker SR, Gibson BG. 2014. Social oral epidemi(olog $)^{2}$ y where next: one small step or one giant leap? Community Dent Oral Epidemiol. 42(6):481-494.

Blinder AS. 1973. Wage discrimination: reduced form and structural estimates. J Hum Resour. 4:436-455.

Burt BA. 2001. Definitions of risk. J Dent Educ. 65(10):1007-1008. 
Centers for Disease Control and Prevention (CDC)/National Center for Chronic Disease Prevention and Health Promotion (NCCDPHP)/Division of Oral Health (DOH), National Health and Nutrition Examination Survey (NHANES). 2009-2010. Oral health examiners manual. Atlanta, GA: CDC

Cobiac LJ, Tam K, Veerman L, Blakely T. 2017. Taxes and subsidies for improving diet and population health in Australia: a cost-effectiveness modelling study. PLoS Med. 14(2):e1002232.

Cobiac LJ, Vos T. 2012. Cost-effectiveness of extending the coverage of water supply fluoridation for the prevention of dental caries in Australia. Community Dent Oral Epidemiol. 40(4):369-376.

Do LG, Spencer AJ. 2016. Oral health of Australian children: the National Child Oral Health Study 2012-14. Adelaide: University of Adelaide.

Kirkpatrick SI, Raffoul A, Maynard M, Lee KM, Stapleton J. 2018. Gaps in the evidence of population interventions to reduce consumption of sugars: a review of reviews. Nutrients. 10(8). pii: E1036.

Lal A, Mantilla-Herrera AM, Veerman L, Backholer K, Sacks G, Moodie M, Siahpush M, Carter R, Peeters A. 2017. Modelled health benefits of a sugarsweetened beverage tax across different socioeconomic groups in Australia: a cost-effectiveness and equity analysis. PLoS Med. 14(6):e1002326.

Larsen K, Merlo J. 2005. Appropriate assessment of neighbourhood effects on individual health: integrating random and fixed effects in multilevel logistic regression. Am J Epidemiol. 161(1):81-88.

Lauridsen J, Christiansen T, Gundgaard J, Häkkinen U, Sintonen H. 2006. Decomposition of socio-economic determination of income-related health inequality by health dimensions. Discussion papers on business and economics No. 8. Odense, Denmark: University of Southern Denmark.

Li C, Yao NA, Yin A. 2018. Disparities in dental health utilization in China. Community Dent Oral Epidemiol. 46(6):576-585.

Mackenbach JP, Kunst AE. 1997. Measuring the magnitude of socio-economic inequalities in health: an overview of available measures illustrated with two examples from Europe. Soc Sci Med. 44:757-771.

Mathur MR, Williams DM, Reddy KS, Watt RG. 2015. Universal health coverage: a unique policy opportunity for oral health. J Dent Res. 94(3 Suppl):3S-5S.

Merlo J, Chaix B, Ohlsson H, Beckman A, Johnell K, Hjerpe P, Råstam L, Larsen K. 2006. A brief conceptual tutorial of multilevel analysis in social epidemiology: using measures of clustering in multilevel logistic regression to investigate contextual phenomena. J Epidemiol Community Health. 60(4):290-297.
Ministry of Health. 2015. Wellington (NZ): New Zealand Government; [updated 2015 Oct 23]. https://www.health.govt.nz/our-work/eating-andactivity-guidelines/current-food-and-nutrition-guidelines.

Moynihan P, Makino Y, Petersen PE, Ogawa H. 2018. Implications of WHO Guideline on Sugars for dental health professionals. Community Dent Oral Epidemiol. 46(1):1-7.

Moynihan PJ, Kelly SA. 2014. Effect on caries of restricting sugars intake: systematic review to inform WHO guidelines. J Dent Res. 93(1):8-18.

National Health and Medical Research Council (NHMRC). 2017. Information paper - water fluoridation: dental and other human health outcomes. Report prepared by the Clinical Trials Centre at University of Sydney. Canberra, Australia: NHMRC

Neumark D. 1988. Employers' discriminatory behaviour and the estimation of wage discrimination. J Hum Resour. 23(3):279-295.

Oaxaca R. 1973. Male-female wage differentials in urban labor markets. Int Econ Rev. 14(3):693-709.

Peres MA, Luzzi L, Peres KG, Sabbah W, Antunes JL, Do LG. 2015. Incomerelated inequalities in inadequate dentition over time in Australia, Brazil and USA adults. Community Dent Oral Epidemiol. 43(6):217-225.

Safiri S, Kelishadi R, Heshmat R, Rahimi A, Djalalinia S, Ghasemian A, Sheidaei A, Motlagh ME, Ardalan G, Mansourian M, et al. 2016. Socioeconomic inequality in oral health behavior in Iranian children and adolescents by the Oaxaca-Blinder decomposition method: the CASPIANIV study. Int J Equity Health. 15(1):143.

Schwendicke F, Thomson WM, Broadbent JM, Stolpe M. 2016. Effects of taxing sugar-sweetened beverages on caries and treatment costs. J Dent Res. 95(12):1327-1332.

Singh A, Harford J, Peres MA. 2018. Investigating societal determinants of oral health: opportunities and challenges in multilevel studies. Community Dent Oral Epidemiol. 46(4):317-327.

Sözmen K, Baydur H, Simsek H, Ünal B. 2012. Decomposing socioeconomic inequalities in self assessed health in Turkey. Int J Equity Health. 11:73.

Spencer AJ, Do LG, Ha DH. 2018. Contemporary evidence on the effectiveness of water fluoridation in the prevention of childhood caries. Community Dent Oral Epidemiol. 46(4):407-415.

Wittekind A, Walton J. 2014. Worldwide trends in dietary sugars intake. Nutr Res Rev. 27(2):330-345. 